\title{
INFLUENCE OF THE FACIAL NERVE ON INTRA-OCULAR PRESSURE*
}

BY

\section{J. GLOSTER}

M.R.C. Ophthalmological Research Unit, Institute of Ophthalmology, University of London

STUDIES of the effects of diencephalic stimulation upon intra-ocular pressure have shown that there are pathways in the central nervous system which, when activated, cause a rise or fall in the pressure in the eye independently of changes in the systemic blood pressure (von Sallmann and Lowenstein, 1955; Gloster and Greaves, 1957). It has already been shown that the cervical sympathetic trunk is the peripheral pathway for central nervous impulses which reduce intra-ocular pressure and that this reduction of pressure is probably due to constriction of the ocular blood supply (Gloster and Greaves, 1958). With regard to rises in intra-ocular pressure induced by diencephalic stimulation, evidence has already been obtained that, in some cases, the rise in pressure in the eye was due to a dilatation of ocular blood vessels (Gloster, 1959), but the peripheral pathway of the nervous impulses responsible for such effects was not known. In view of the accepted association between central nervous factors and the occurrence of attacks of acute glaucoma, it was clearly important that an attempt should be made to discover peripheral nervous pathways mediating rises in intraocular pressure.

Perkins (1957) demonstrated that, in the rabbit, stimulation of the ophthalmic division of the trigeminal nerve evokes a large rise in intra-ocular pressure, but he was of the opinion that this effect was of the nature of an axon reflex and not the result of activity of true efferent fibres emanating from the central nervous system and influencing the vascular system of the eye.

In the present study an investigation has been made of the possibility that the peripheral pathway for centrally-induced rises in intra-ocular pressure is in the parasympathetic component of the seventh cranial nerve. Briefly, the work has proceeded along the following lines. Rises in intra-ocular pressure, of a type believed to have a vascular causation, were elicited by diencephalic stimulation in cats and the effect of dividing the facial nerve at the internal auditory meatus was studied; the results suggested that the seventh nerve was the peripheral pathway of these rises in intra-ocular pressure but were not conclusive. The next step was to stimulate the facial

* Received for publication August, 11, 1960. 
nerve at the point where the greater superficial petrosal nerve, which carries a number of the parasympathetic fibres, leaves the main part of the nerve. In both cats and rabbits this procedure led to a rise in intra-ocular pressure. The fibres of the facial nerve were then stimulated in the floor of the fourth ventricle in rabbits; this also gave an increase in intra-ocular pressure, which was abolished after peripheral destruction of the facial nerve at the point of emanation of the greater superficial petrosal nerve. In addition, the effects of some drugs upon the increase in intra-ocular pressure caused by stimulation of the facial nerve were studied.

\section{Methods}

All experiments were carried out under general anaesthesia, using chloralose ( $80-120 \mathrm{mg} . / \mathrm{kg}$. intravenously) after ether induction for cats, and urethane $(1 \cdot 75-$ $2.5 \mathrm{~g} . / \mathrm{kg}$. intravenously) for rabbits. Intra-ocular pressures and femoral arterial pressure were recorded as previously described by Perkins (1957). In a considerable number of animals the blood pressure was rather low, but could often be improved, temporarily at least, by the intravenous injection of Dextran $20-40 \mathrm{ml}$.

\section{(a) Diencephalic Stimulation and Intra-cranial Division of the Facial Nerve}

Cats were prepared for diencephalic stimulation, using the stereotaxic procedure adopted by Gloster and Greaves (1957), and the technique of stimulation was that described previously (Gloster, 1959). In addition, a trephine-hole was made in the roof of the posterior fossa and enlarged with bone-forceps, and cerebellum was removed until the internal auditory meatus could be seen. The electrode was then inserted into the diencephalon in a position likely to give the type of rise in intra-ocular pressure to be investigated, and a stimulus was applied for 15 seconds. (This short duration of stimulation was necessary since it was found that with more prolonged stimulations the response could not be obtained again until a period of 15 to 20 minutes had elapsed.) If necessary, the position of the electrode was altered until the required rise in intra-ocular pressure was obtained. The response to two or three stimulations was recorded and then the seventh and eighth cranial nerves were divided at the internal auditory meatus. This procedure could be carried out without displacing the diencephalic electrode because in the cat the tentorium cerebelli is ossified and the contents of the middle and anterior cranial fossae are not affected by small manipulations in the posterior fossa. After the seventh nerve had been divided the diencephalic stimulation was repeated several times without altering the position of the electrode. After the animal had been killed the brain was removed and histological sections prepared to demonstrate the position of the stimulating electrode (Gloster and Greaves, 1957).

\section{(b) Stimulation of the Greater Superficial Petrosal Branch of the Facial Nerve}

When a cat was used, the head was mounted in the stereotaxic instrument and a longitudinal incision was made in the scalp and a second incision at right angles to this running down posterior to the right external ear. The soft tissues were 
reflected downwards to expose the right facial nerve as it emerged from the stylomastoid foramen. With a small electrically driven dental burr, bone was carefully removed to expose the nerve in the facial canal, starting at the stylomastoid foramen and following the nerve centrally until it passed medially to the point at which the greater superficial petrosal nerve leaves the main trunk of the facial nerve. It was possible to open up the facial canal in this way sufficiently far for a straight electrode to be inserted so that its tip lay at the origin of the greater superficial petrosal nerve. The electrode used was a stainless steel needle, $0.33 \mathrm{~mm}$. external diameter, insulated by three layers of Damarda lacquer L.2138; the insulation was scraped off the end of the needle for a distance of $1 \mathrm{~mm}$. This electrode was held in position by suitable supporting clamps fixed to the stereotaxic instrument. The tip of a second similar electrode was then inserted into the opened facial canal so that its tip lay about $2 \mathrm{~mm}$. externally to the tip of the first electrode. The stimulating current was passed between these two electrodes in order to apply as localized a stimulus as possible to the greater superficial petrosal nerve.

When a rabbit was used, the head was mounted in a head-holder and an incision was made in the skin below and posterior to the root of the pinna. The soft tissues were divided and reflected until the facial nerve was seen as it emerged from the stylomastoid foramen. Part of the bone forming the external auditory meatus was exposed and excised, and after the bone covering the facial canal had been removed an opening was made into the middle ear cavity. There was only a very thin layer of bone which separated the nerve from the middle ear cavity and this was easily removed so that the nerve could be seen running along the medial wall of the cavity. After removing the auditory ossicles the nerve could be seen clearly at the point where it disappeared into the dense bone of the petrous temporal; from this point the canal ran quite straight for a distance of $3-4 \mathrm{~mm}$. to the origin of the greater superficial petrosal nerve. A straight electrode could then be inserted into this part of the canal so that its tip lay beneath the origin of the greater superficial petrosal nerve; the tip of the electrode automatically reached this point and could not be pushed further because the facial canal bends sharply medially at the origin of the greater superficial petrosal nerve. Since the tip of the electrode was unable to move further and since the electrode was supported for a distance of 3-4 mm. in the facial canal, it was only necessary to apply gentle inward pressure to keep the electrode firmly in position. This pressure was applied by fastening a small piece of perspex to the electrode about $2 \mathrm{~cm}$. from its tip and attaching a small looped rubber band to this; the loops of the rubber band were then attached to the soft tissues by means of sutures, the necessary amount of tension in these anchoring sutures being easily adjusted. In some experiments, a concentric bipolar electrode, $0.45 \mathrm{~mm}$. external diameter, was fixed in the facial canal, but unfortunately the canal was often not large enough to admit this type of electrode, and a single electrode had to be used in conjunction with an indifferent electrode in the rectum of the rabbit.

\section{(c) Stimulation of the Facial Nerve in the Floor of the Fourth Ventricle}

In the rabbit, the nucleus of the seventh cranial nerve is situated in the ventrolateral regions of the pons, and the axons emanating from the nucleus run dorsally and medially in a fairly well-defined band. On each side of the mid-line, this band 
of axons passes around the medial and then the dorsal aspect of the nucleus of the sixth nerve, so that just beneath the floor of the fourth ventricle the fibres of the nerves on the two sides approach each other closely. It was thought that stimulation in this area should therefore evoke a bilateral effect on the intra-ocular pressure. The problem was how to place the stimulating electrode in a suitable position, having regard to the fact that the use of standard stereotaxic techniques is difficult in the rabbit. By cutting frontal sections of the heads of dead animals it was found that the point at which the facial nerve disappeared into the petrous temporal bone (which could be seen from the middle ear cavity as described in the dissection above) gave a good indication of the antero-posterior and vertical levels of the point where the fibres of the right and left facial nerves come into juxtaposition beneath the floor of the fourth ventricle. The head of the animal was mounted in a head-holder, and the latter was fixed in the stereotaxic instrument, taking care that the longitudinal axis of the head corresponded with the anteroposterior axis of the instrument. The stereotaxic instrument was used not so that the tip of the electrode could be orientated by the standard techniques, but merely to provide a suitable electrode carrier by means of which the tip of the electrode could be moved accurately in directions parallel to the longitudinal and transverse axes of the head of the animal. After the dissection described above had been carried out, the position of the point at which the facial nerve disappeared from the middle ear cavity into the petrous temporal bone could be determined with regard to vertical and antero-posterior co-ordinates; it was then only necessary to displace the tip of the electrode medially to the median plane of the head in order to get it into the correct position, i.e. just beneath the floor of the fourth ventricle at the point where the fibres of the right and left facial nerves come close together. The sagittal suture was used as a guide to the median plane and a burr-hole, $1.5 \mathrm{~mm}$. in diameter, was drilled in the vault of the skull through which the electrode could be inserted. It was found necessary to modify the mounting of the electrode carrier on the stereotaxic instrument so that the electrode, instead of being inserted vertically, was inserted with a tilt of $30^{\circ}$, the tip of the electrode being anterior to its other end. This modification was required because, with vertical insertion of the electrode, the burr-hole in the skull had to be drilled at the point of confluence of some of the larger venous sinuses, a procedure which led to uncontrollable haemorrhage. After the animal had been killed, the brain was removed and the usual histological procedures were carried out in order to verify the position of the electrode.

\section{Results}

\section{(a) Diencephalic Stimulation before and after Division of the Facial Nerve}

Fig. 1 (opposite) illustrates an experiment in which diencephalic stimulation gave a rise in pressure in the right eye (Stim. 1) which could not be attributed solely to the small accompanying rise in blood pressure for reasons which have already been given (Gloster, 1959). Between the two stimulations shown in Fig. 1, the facial nerve was divided at the internal auditory meatus, and in the subsequent stimulation it can be seen that the rise in right intra-ocular 

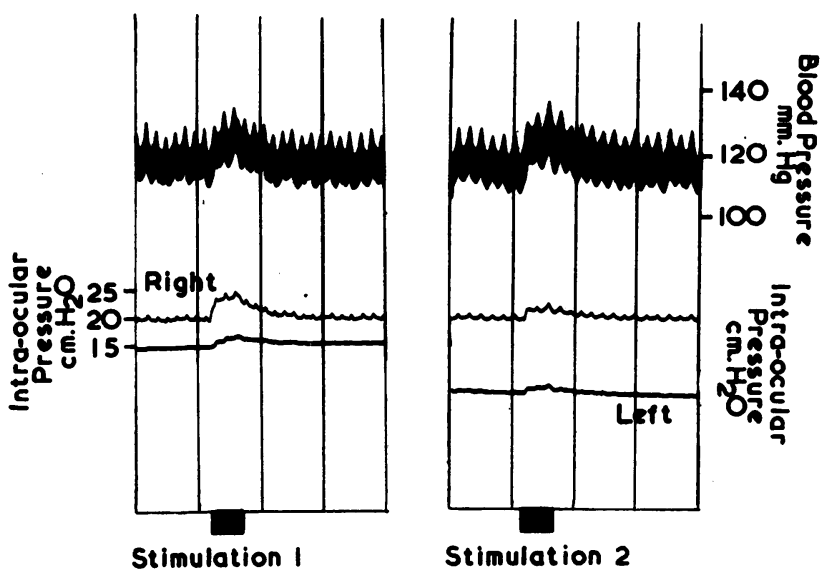

FIG. 1.-Diencephalic stimulation in a cat. Electrode position $12 \mathrm{~mm}$. anterior to inter-aural line, $2 \mathrm{~mm}$. to right of mid-line, at level of junction of thalamus and hypothalamus. Between the two stimulations illustrated, the seventh and eighth cranial nerves were divided at the internal auditory meatus. Stimulus $3.2 \mathrm{v}, 12.5 \mathrm{msec}$., $10 \mathrm{c} / \mathrm{s}$.

(The position of the record of left intra-ocular pressure was altered between the two stimulations shown.)

In this and the succeeding illustrations, Blood Pressure $=$ femoral arterial pressure, and the interval between successive vertical lines indicates a period of $60 \mathrm{sec}$.

pressure was considerably less, although the rise in blood pressure and the small increase in pressure in the left eye were not significantly different from the corresponding responses in the first stimulation. In a second cat, an almost identical result was obtained. There was a less marked diminution of the rise in intra-ocular pressure in a third cat, and in a fourth cat section of the facial nerve did not appear to have any influence on the response.

\section{(b) Stimulation of the Facial Nerve at the Origin of the Greater Superficial Petrosal Nerve}

If the facial nerve had not been divided at the stylomastoid foramen, stimulation caused rhythmic twitching of the muscles supplied by the motor fibres of the facial nerve, i.e. muscles of the face, scalp, the platysma, and the eyelids. This twitching occurred on the side of stimulation and stopped instantly on cessation of stimulation. Such motor effects were absent if the facial nerve had been divided at the stylomastoid foramen, but it was found that in some animals there was also a fine bilateral nystagmus; this was considered to be due to a slight spread of stimulation to the vestibular division of the eighth nerve which, after entering the internal auditory meatus with the facial nerve, lies extremely close to the latter for a short distance. The vestibular origin of the nystagmus was proved in one animal, since, during the dissection a small opening was made into the lateral semicircular canal, and when the tip of the electrode was placed in this aperture and a stimulating current applied, bilateral nystagmus (with no other 
muscular effects) was obtained. These motor reactions sometimes produced complex changes in the pressure in both eyes through direct pressure on the globe of the extra-ocular muscles or the muscles of the eyelids, as can be seen in Fig. 2, which illustrates the response obtained in a cat. In this animal stimulation on the right side at the origin of the greater superficial petrosal nerve produced a small rise in blood pressure, together with slight muscular twitching of the right facial muscles and bilateral nystagmus. In the right eye, there was a small abrupt fall in pressure at the beginning of stimulation, followed by a rise of about $2.5 \mathrm{~cm}$. saline, and the pressure returned to normal in about 30 seconds after cessation of stimulation. In the left eye there was a smaller rise in pressure and, in contrast to the right eye, there was an abrupt fall in pressure on cessation of stimulation.

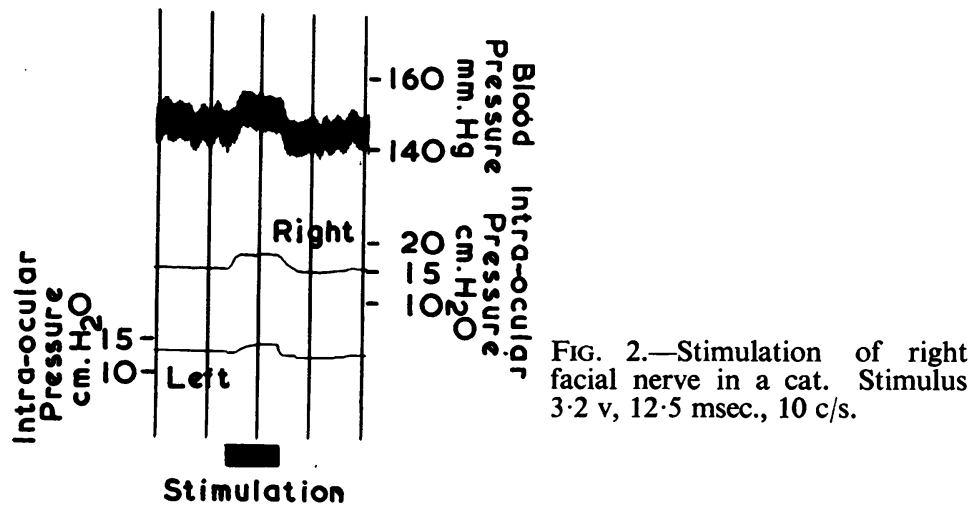

Fig. 3 shows the results of three successive stimulations of the right facial nerve in another cat. In the first stimulation, there was some twitching of the muscles of the right side of the face, but no nystagmus, and the right pupil dilated very slightly (about $1 \mathrm{~mm}$.); the figure shows that there was no significant change in blood pressure, a definite increase in right intra-ocular
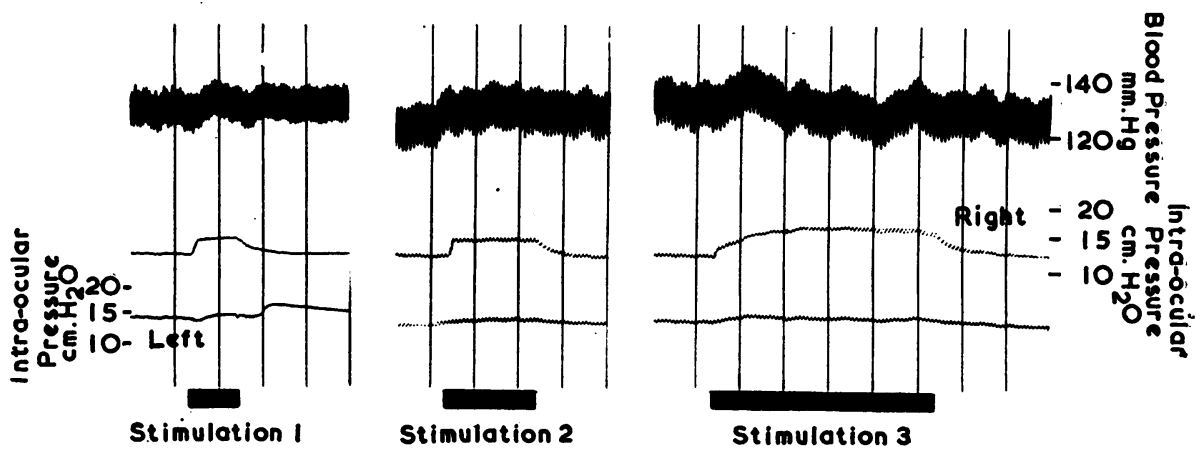

Fig. 3.-Stimulation of right facial nerve in a cat. Stimulus $4 \mathrm{v}, 12.5 \mathrm{msec} ., 10 \mathrm{c} / \mathrm{s}$. 
pressure, and some rather complex changes in pressure in the left eye. The latter could perhaps be due to changes in the tone of the extra-ocular muscles induced by a small spread of stimulation to the vestibular branch of the eighth cranial nerve, and to eliminate all possibility that striated muscular activity was responsible for these changes in intra-ocular pressure, Flaxedil (gallamine triethiodide) was injected intravenously. Between the first and second stimulations in this animal, Flaxedil was given in a dose of $2 \mathrm{mg} . / \mathrm{kg}$.; this caused cessation of spontaneous respiration and the animal was respired artificially by means of a pump. During the first 60 seconds of the second stimulation, there was still a very slight twitching of the vibrissae of the right side and although barely perceptible these oscillations showed that there was still some striated muscular activity. Accordingly, an additional dose $(1 \mathrm{mg} . / \mathrm{kg}$.) Flaxedil was given, and at the third stimulation there was not the slightest visible evidence of muscular activity. Fig. 3 shows that after the abolition of striated muscular effects there were no significant changes in the left intra-ocular pressure. In all three stimulations, the blood pressure showed no sustained change during stimulation but there was a rise in pressure of about $3 \mathrm{~cm}$. saline in the right eye; the magnitude of this effect was undiminished after the abolition of all striated muscular activity with Flaxedil, although the character of the response was perhaps altered slightly with regard to the initial rate of elevation of pressure. It is to be noted that at the third stimulation which lasted for 5 minutes the rise in pressure in the right eye was maintained for the full period of stimulation. It was also observed during the third stimulation that there was a minute dilatation of the right pupil, that there was not the slightest visible evidence of any movement of the nictitating membrane, and that there was some lacrimation in the right eye (but not in the left) and also some salivation.

Fig. 4 shows the effect of a prolonged stimulation in another cat. Just before this stimulation, Flaxedil was given to eliminate the possibility that any rise in intra-ocular pressure was due to striated muscular activity. Before giving Flaxedil, the responses of the blood pressure and intra-ocular

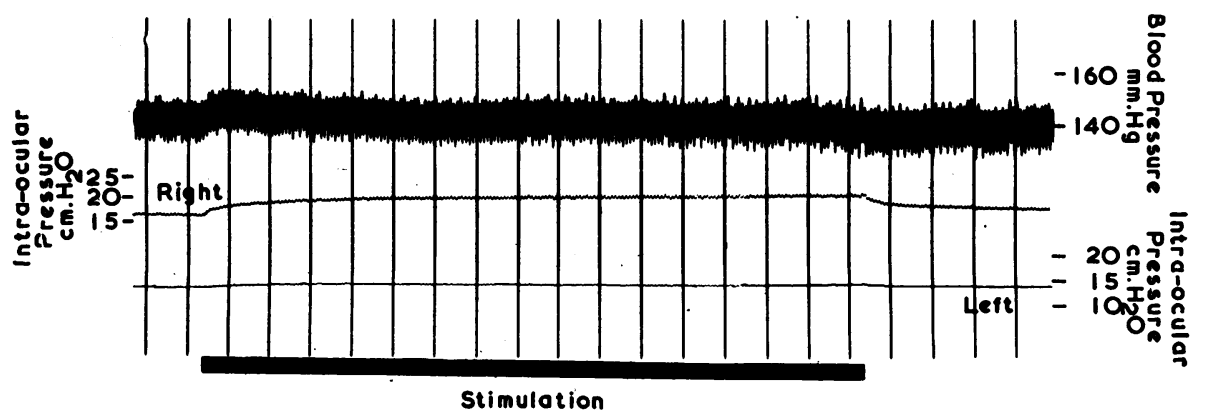

Fig. 4.-Stimulation of right facial nerve in a cat. Stimulus $3.2 \mathrm{v}, 12.5 \mathrm{msec} ., 10 \mathrm{c} / \mathrm{s}$. 
pressures were those shown in Fig. 2. From Fig. 4 it will be noted that with a stimulation which persisted for 16 minutes, the intra-ocular pressure rose gradually all the time, although the systemic blood pressure remained almost constant. The final rise in pressure in the right eye amounted to about $6 \mathrm{~cm}$. saline, and at the cessation of stimulation the pressure began to fall immediately towards its original value. Stimulation also produced lacrimation in the right eye of this animal and there was slight salivation.

The technique described was used on five cats and similar effects were obtained in all animals. In two animals, the effects were less definite, but in another cat the rise in intra-ocular pressure was unusually large, amounting to $14 \mathrm{~cm}$. saline. Lacrimation in the eye on the stimulated side and slight salivation were obtained in all five animals.

In rabbits, the responses obtained were generally similar to those described for cats. Fig. 5 shows the effect of stimulation of the right facial nerve at the origin of the greater superficial nerve. In this animal there was no nystagmus, but both eyes moved slightly at the beginning of stimulation and then remained still; there were no other motor effects (the facial nerve having been divided at the stylomastoid foramen), no change in the rate or rhythm of respiration, and no alteration in the size of the pupils. In the right eye there was marked lacrimation and salivation was also produced.

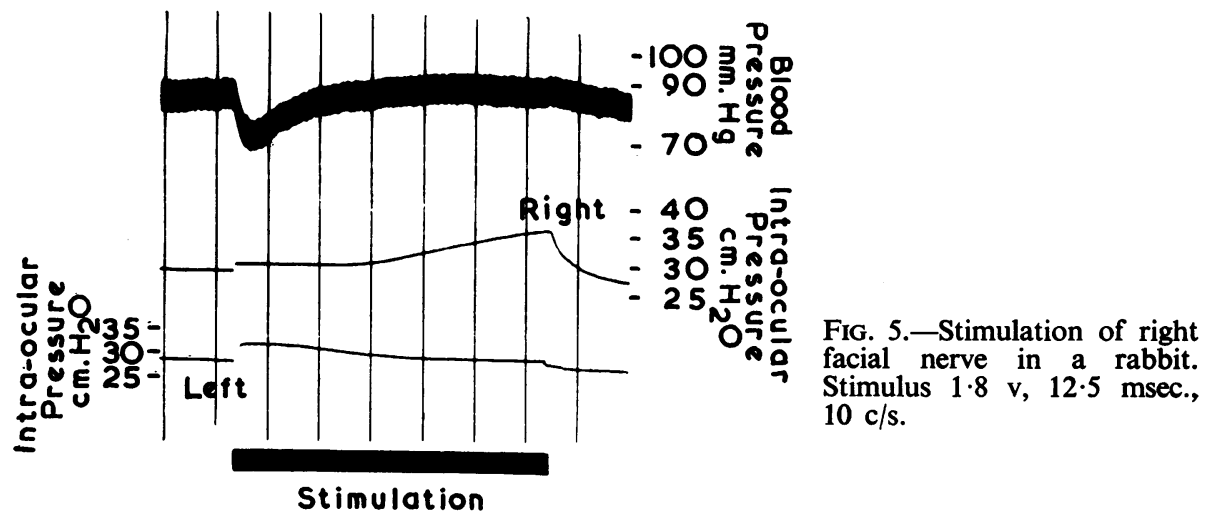

There was a transient fall in blood pressure of about $15 \mathrm{~mm} . \mathrm{Hg}$. The right intra-ocular pressure showed an initial abrupt elevation and then a more gradual rise; as soon as stimulation ceased the pressure began to fall. In the left eye the pressure rose rapidly as soon as stimulation began, but then began to fall slowly. The changes in pressure were ascribed in the left eye wholly and in the right eye partly to changes in tone of the extra-ocular muscles due to slight spread of the stimulation to the vestibular division of the eighth cranial nerve.

On occasions, these muscular effects produced highly complex alterations in intra-ocular pressure. In the animal shown in Fig. 6 (opposite), the first stimulation caused definite nystagmus in both eyes, but there were no 
other motor effects, no respiratory change, and no pupillary activity. Lacrimation occurred in the right eye, but salivation was not noticed. There was a transient fall in blood pressure, while the responses of the intraocular pressure are best judged from the illustration. In order to eliminate changes in intra-ocular pressure attributable to muscular pressure on the eyeballs, Flaxedil $(2 \mathrm{mg} . / \mathrm{kg}$.) was injected intravenously and artificial respiration started. On repeating the stimulation, there was no fall in the blood pressure (this modification of the response after giving Flaxedil was unusual) and no change in the left intra-ocular pressure, but a rise in the pressure of about $8 \mathrm{~cm}$. saline occurred in the right eye; there was now not the slightest movement of either eye during stimulation.
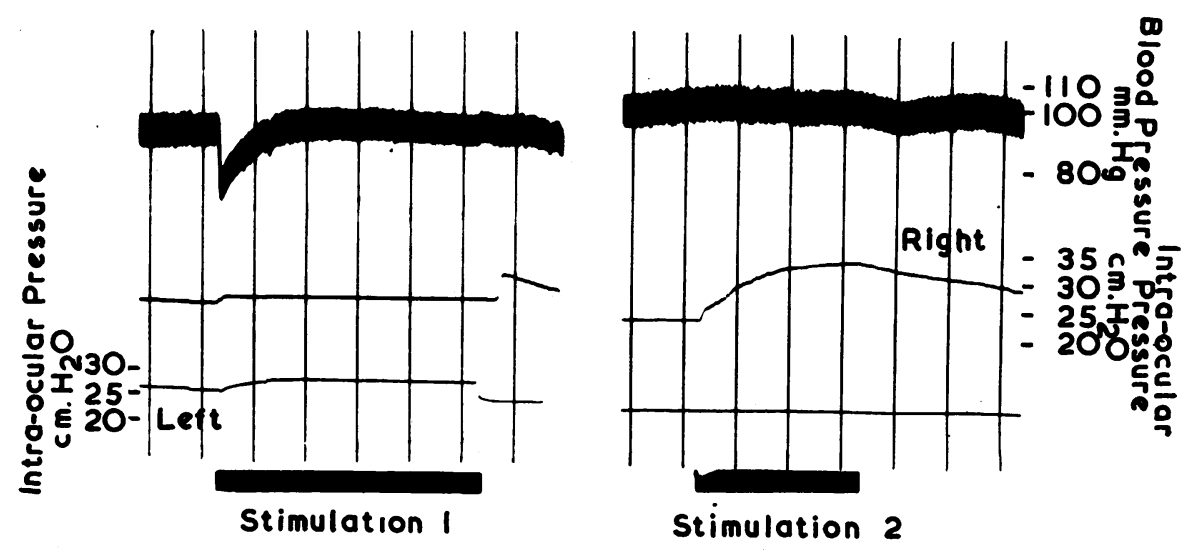

FIG. 6.-Stimulation of right facial nerve in a rabbit. Between the two responses shown Flaxedil was injected intravenously. Stimulus $2 \cdot 2 \mathrm{v}, 12 \cdot 5 \mathrm{msec}$., $10 \mathrm{c} / \mathrm{s}$.

The effects of stimulation of the facial nerve in three other rabbits are illustrated in Figs 7, 8, and 9. The rise in right intra-ocular pressure shown in Fig. 7 was accompanied by lacrimation, but there was no nystagmus nor any other motor effect, and the animal had not been given Flaxedil.

Fig. 7.-Stimulation of right facial nerve in a rabbit. Stimulus $1 \cdot 3 \mathrm{v}, 12 \cdot 5 \mathrm{msec}$., $10 \mathrm{c} / \mathrm{s}$.

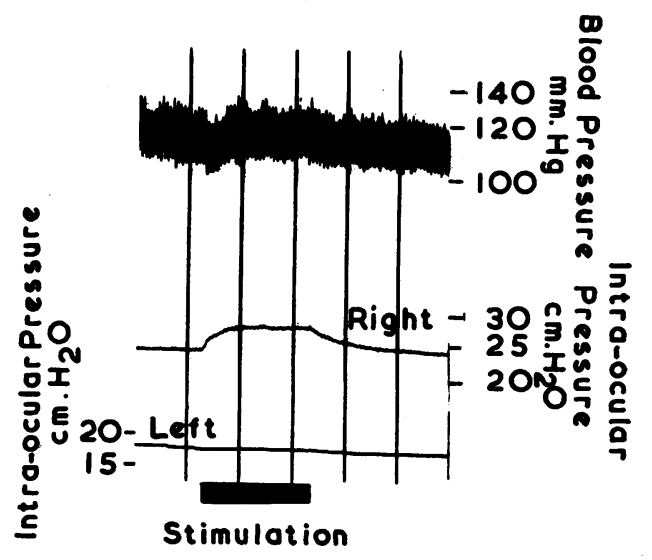


The response shown in Fig. 8 differs from the others in that it was obtained by using a concentric bipolar electrode in the facial canal. In this animal stimulation produced nystagmus and lacrimation in the right eye only, but there was no salivation or pupillary change. Before the stimulation illustrated, Flaxedil had been given to eliminate striated muscular activity, but a rise in the pressure in the right eye was clearly obtained. That striated muscular activity has nothing to do with the production of this rise in intraocular pressure was proved also in this rabbit because, when the effect of Flaxedil had worn off, stimulation again produced nystagmus in the right eye, but the rise in pressure was less than when the eye was immobile.

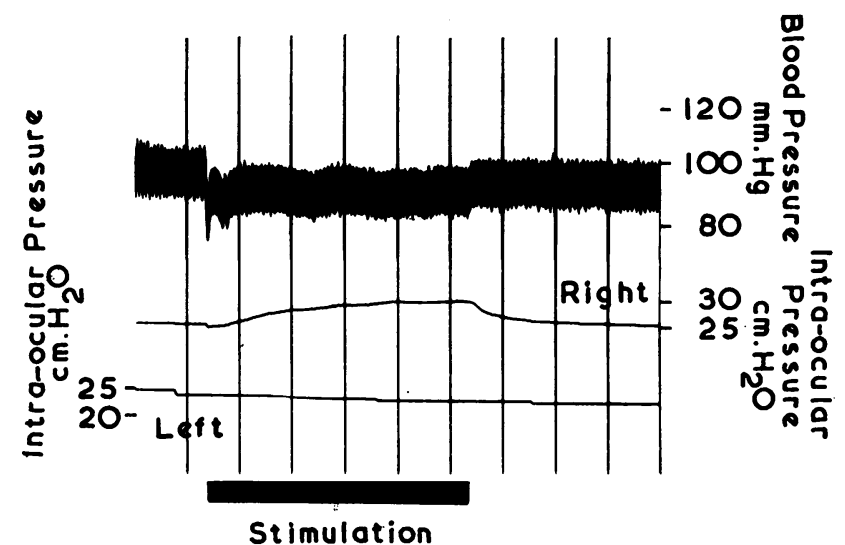

FIG. 8.-Stimulation of right facial nerve in a rabbit. Stimulus $4 \mathrm{v}$, $12.5 \mathrm{msec}$., $10 \mathrm{c} / \mathrm{s}$.

Fig. 9 shows a rise in intra-ocular pressure in the right eye which was obtained in another rabbit. The sole motor effect was an extremely fine oscillation of the right eye, perceptible only as a minute movement in the

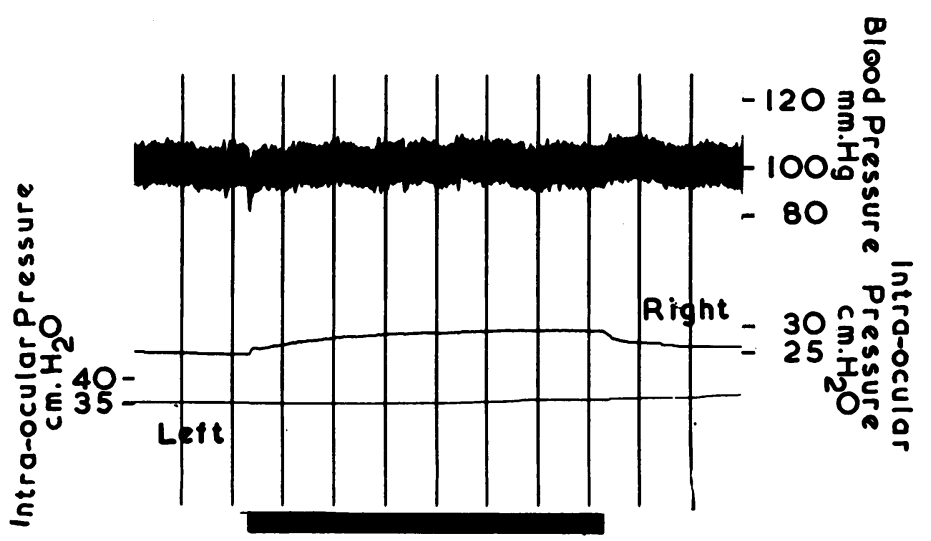

Stimulation

FIG. 9.-Stimulation of right facial nerve in a rabbit. Stimulus $1.7 \mathrm{v}$, $12.5 \mathrm{msec}$., $10 \mathrm{c} / \mathrm{s}$. 
reflection of a point of light from the lower part of the cornea. Flaxedil was not given before the response illustrated, but when given later it did not abolish the rise in intra-ocular pressure. The response shown was accompanied by lacrimation in the right eye, but salivation was absent and neither pupil changed in size.

From the responses illustrated above, it can be seen that, if the effects of muscular pressure on the globe are insignificant or have been eliminated, stimulation of the facial nerve in the rabbit causes a rise in pressure in the eye on the same side as the stimulating electrode. There is sometimes a small and fairly rapid initial rise in pressure which is succeeded by a more gradual rise. The rise in pressure is maintained until the end of stimulation, after which there is a return to normal pressure over a period of a few minutes; the fall at the end of stimulation was never abrupt. Although the responses varied in some respects from animal to animal, there were sufficient features in common to make it clear that the same type of rise in intra-ocular pressure could be obtained repeatedly.

The technique described for stimulation of the facial nerve was applied in 22 rabbits, using bipolar stimulation in five, and unipolar stimulation in the remaining seventeen. The voltage applied varied between 3 and $4.5 \mathrm{v}$ with bipolar stimulation and between 0.9 and $3.2 \mathrm{v}$ with unipolar stimulation; the pulse width was $12.5 \mathrm{msec}$. and the pulse frequency $10 \mathrm{c} / \mathrm{s}$. Of the 22 rabbits, five did not give a characteristic rise in intra-ocular pressure, ten gave a definite rise in pressure of 1 to $3 \mathrm{~cm}$. saline, and seven gave rises in pressure of more than $4 \mathrm{~cm}$. saline, the greatest being $11 \mathrm{~cm}$. These figures give only an approximate indication of the incidence of weak and strong responses because in a number of stimulations the intra-ocular pressure was still rising slowly at the end of stimulation. The characteristic rise in pressure occurred only in the eye on the same side as the stimulating electrode. Lacrimation in the eye on the side of stimulation was produced in all 22 rabbits, although in two it was very slight, but salivation was observed less frequently.

\section{(c) Stimulation of the Facial Nerve in the Floor of the Fourth Ventricle}

In this series of experiments the intention was to place the tip of the stimulating electrode 0.5 to $1 \mathrm{~mm}$. below the floor of the fourth ventricle, exactly in the mid-line of the brain in a region where the fibres emanating from the right and left facial nerve nuclei come into juxtaposition. Histological preparations showed that this position was achieved in five out of fourteen rabbits in this series; one of the stained sections is reproduced in Fig. 10 (overleaf). In a further five rabbits, the position of the electrode was similar except for a deviation of 0.5 to $1 \mathrm{~mm}$. to one side of the mid-line. In three rabbits, the electrode was in the mid-line but not at the correct height; in one the tip had not penetrated the roof of the fourth ventricle and lay in 


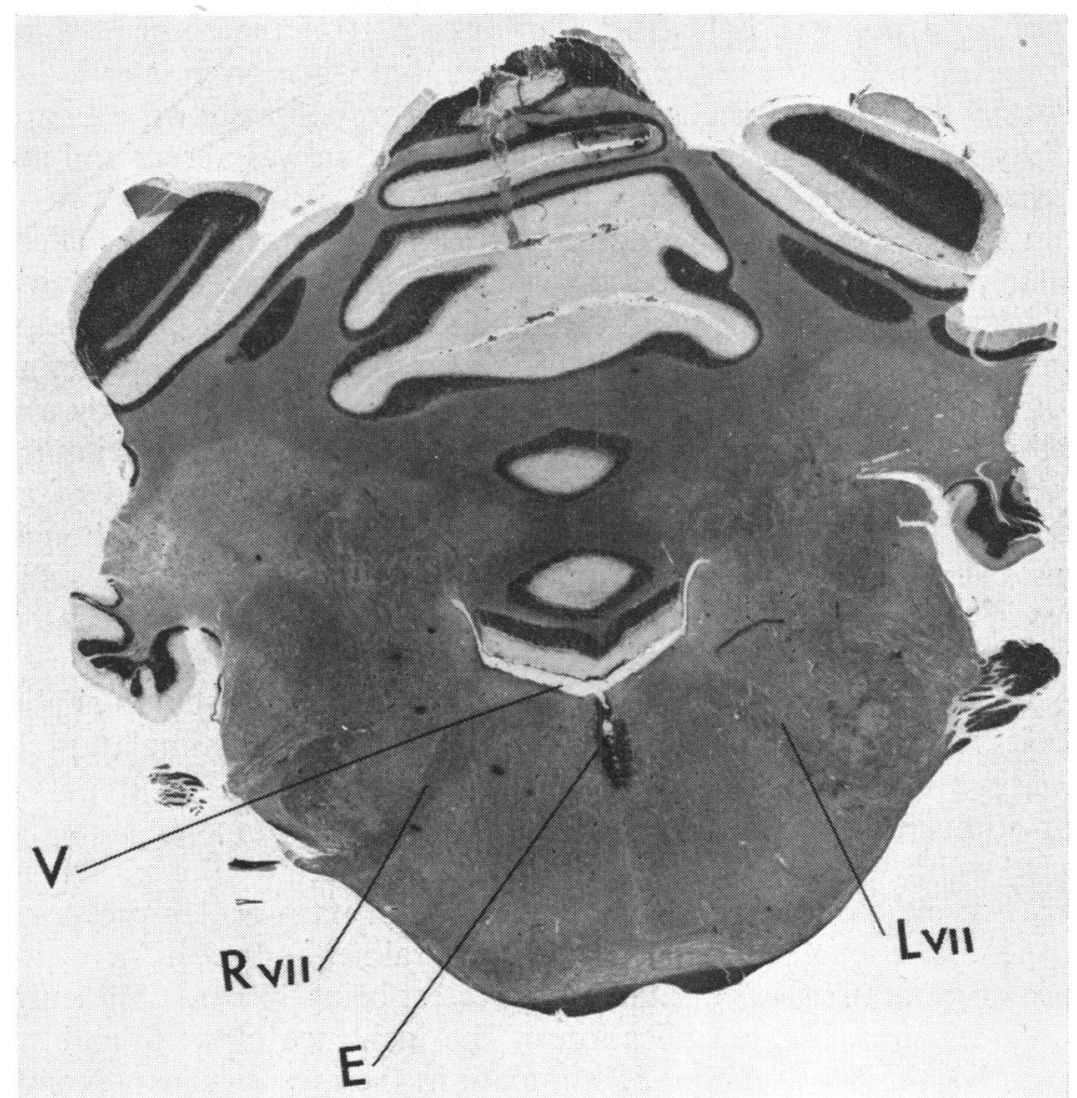

FIG. 10.-Section through cerebellum and pons of a rabbit, stained with haemotoxylin andeosin, to show position of tip of stimulating electrode in floor of fourth ventricle.

$$
\begin{aligned}
E & =\text { Position of tip of electrode } \\
R \text { VIII } & =\text { Fibres of right facial nerve } \\
\text { L VII } & =\text { Fibres of left facial nerve } \\
\text { V } & =\text { Fourth ventricle. }
\end{aligned}
$$

the extreme ventral part of the cerebellum, and in two the tip of the electrode was about $2 \mathrm{~mm}$. below the floor of the fourth ventricle. In the remaining rabbit, the tip of the electrode was about $1 \mathrm{~mm}$. from the mid-line and about $1.5 \mathrm{~mm}$. below the required level.

In all five rabbits in which the tip of the electrode was exactly in the required position, stimulation gave a rise in pressure in both eyes. A marked response is seen in Fig. 11 (opposite); there was scarcely any change in the systemic blood pressure, but the intra-ocular pressure began to rise in both eyes at the commencement of stimulation and continued to do so, until the cessation of stimulation when the pressures in both eyes fell gradually to their original levels. During stimulation, the vibrissae on the right side were oscillating slightly, indicating that the motor fibres of the facial nerve 


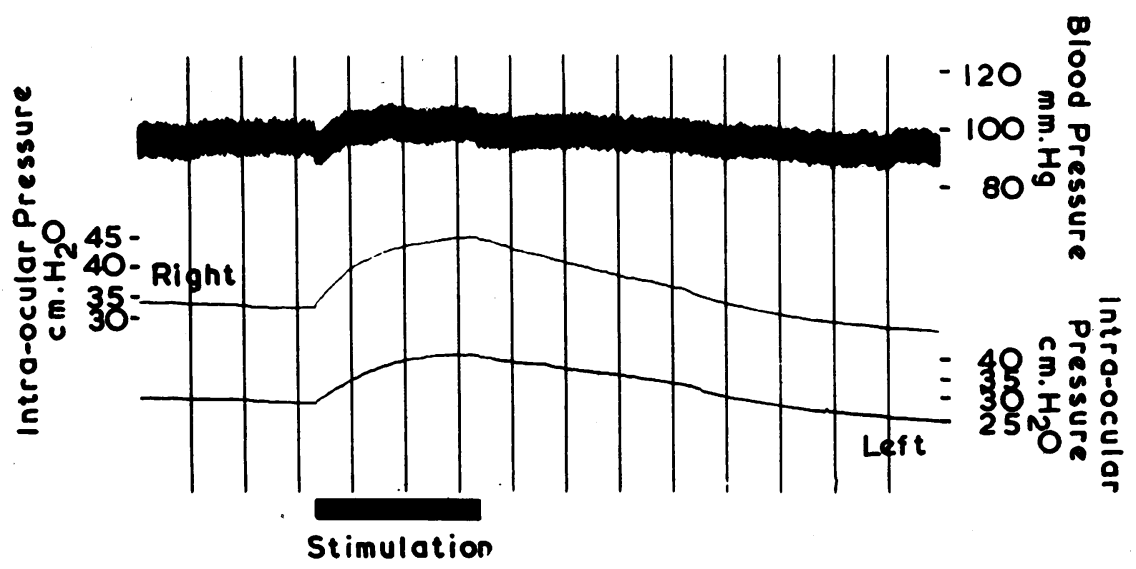

Fig. 11.-Stimulation in mid-line in floor of fourth ventricle of a rabbit. Stimulus $2 \cdot 8 \mathrm{v}$,

$12.5 \mathrm{msec} ., 10 \mathrm{c} / \mathrm{s}$.

were being stimulated, and much weaker movements were visible on the left side; these movements stopped abruptly when stimulation ceased. There were no other motor effects and no movements of the nictitating membranes, so that there was a complete absence of any evidence to suggest that smooth muscular activity contributed to the rise in intra-ocular pressure. In another stimulation, after Flaxedil had been given, similar rises in intra-ocular pressure were obtained, and this observation demonstrated that striated muscular contractions could have nothing to do with the elevation of pressure. During the stimulation illustrated in Fig. 11, lacrimation was observed in both eyes and there was some salivation, but the pupils did not change in size. The rises in intra-ocular pressure in this animal were the largest observed in this series of experiments, the bilateral effects in the remaining rabbits being considerably smaller.

In five animals, the tip of the electrode lay slightly to one side of the midline; in one of these rabbits there was no rise in pressure in either eye during stimulation and in another there was a very small bilateral rise. In the three remaining animals, stimulation caused a definite rise in pressure in the eye on the same side of the mid-line as the tip of the electrode; such an effect is illustrated in Fig. 12 (overleaf), from which it is clear that the response of the intra-ocular pressure is remarkably similar to that obtained by stimulation in the facial canal in other rabbits. The rise in intra-ocular pressure shown in Fig. 12 was not accompanied by any motor effects, because the facial nerve on the left side had been divided at the stylo-mastoid foramen; lacrimation was observed in the left eye and there was no change in the size of the pupils.

In some experiments, after obtaining a rise in pressure in one or both eyes by stimulation in the floor of the fourth ventricle, the facial nerve was destroyed at the point in the facial canal where the greater superficial 


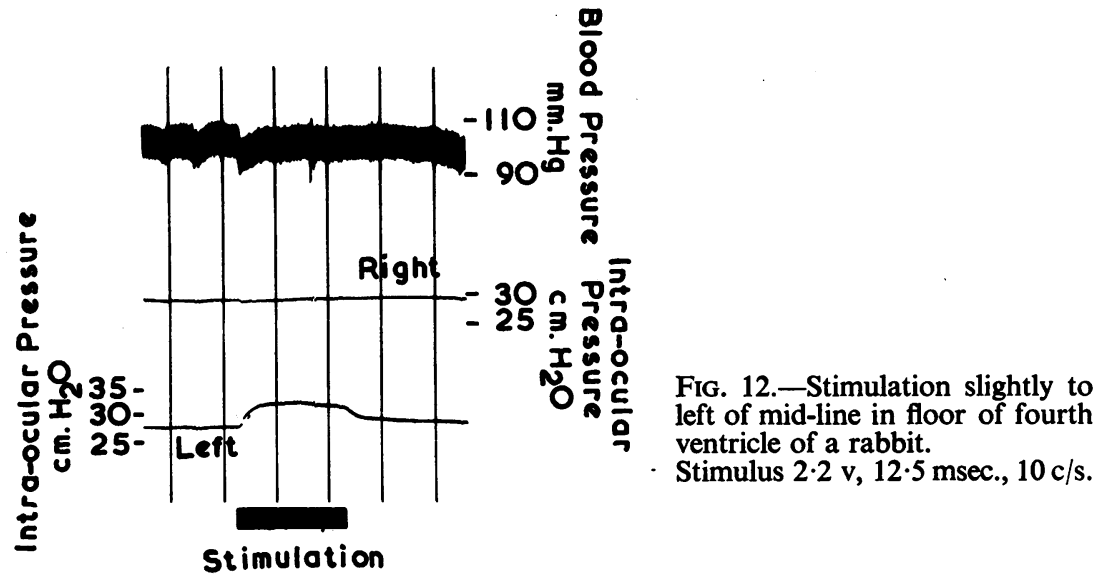

petrosal nerve leaves the main trunk of the nerve. This was done by inserting a hypodermic needle into the canal from the middle ear cavity and rotating the needle about its axis repeatedly; at the conclusion of the experiment, when the animal had been killed, the facial canal was opened in order to verify that all connexion between the greater superficial petrosal nerve and the facial nerve had been destroyed. This procedure was carried out on five rabbits, and two such experiments are illustrated in Fig. 13 and in Fig. 14 (opposite).

During the two stimulations shown in Fig. 13, the only motor effect was a slight nystagmus of the right eye. There was no change in respiration, the pupils remained unchanged in size and salivation occurred. The first stimulation caused little change in the systemic blood pressure, lacrimation in both eyes, and a bilateral rise in intra-ocular pressure. The greater superficial petrosal nerve was then destroyed at its origin on the left side and in the subsequent stimulation lacrimation was obvious in the right eye
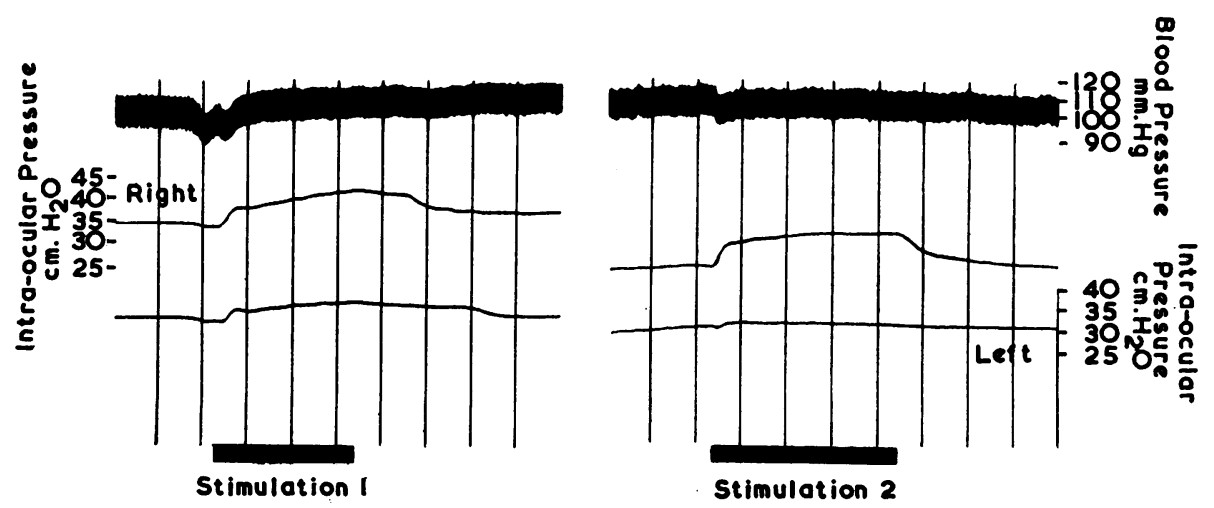

FIG. 13.-Stimulation in mid-line in floor of fourth ventricle of a rabbit. Between the two stimulations shown the left facial nerve was destroyed at the origin of the greater superficial petrosal nerve. Stimulus $3.5 \mathrm{v}, 12.5 \mathrm{msec}$., $10 \mathrm{c} / \mathrm{s}$. 
only; as the illustration shows, the second stimulation evoked scarcely any change in blood pressure and there was no significant change in the left intra-ocular pressure, while the pressure in the right eye rose as in the previous stimulation.

In the experiment shown in Fig. 14, the tip of the electrode lay in the floor of the fourth ventricle slightly to the left of the mid-line, and it can be seen that stimulation caused no change in the right intra-ocular pressure, although some lacrimation was observed in this eye. During the first stimulation, lacrimation also occurred in the left eye and there was a small but definite rise in the intra-ocular pressure in the left eye. Between the two stimulations shown in Fig. 14 the origin of the greater superficial petrosal nerve was destroyed on the left side and stimulation was applied again in the floor of the fourth ventricle. The responses of the blood pressure and intra-ocular pressure in the right eye were unchanged after destruction of the petrosal nerve on the left side, but the small rise in pressure in the left eye gave place to a fall in pressure.
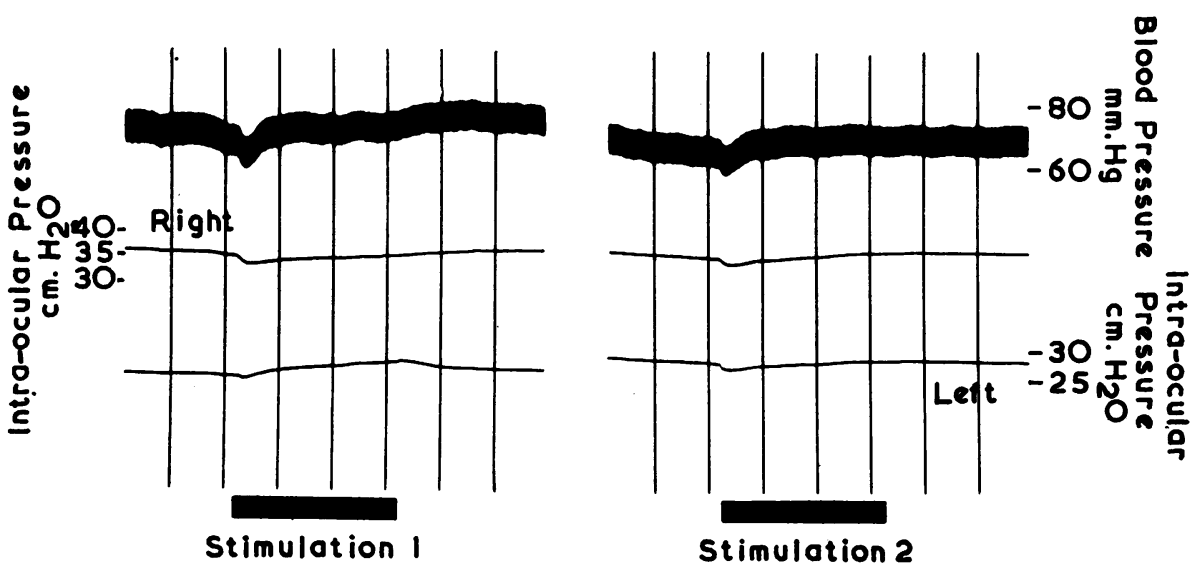

Fig. 14.-Stimulation slightly to left of mid-line in floor of fourth ventricle in a rabbit.

Between the two stimulations shown the left facial nerve was destroyed at the origin of the greater superficial petrosal nerve. Stimulus $2.8 \mathrm{v}, 12.5 \mathrm{msec} ., 10 \mathrm{c} / \mathrm{s}$.

Similar results were obtained in four out of five experiments of this type, a rise in pressure in one eye evoked by stimulation in the fourth ventricle being abolished by division of the greater superficial petrosal nerve. In the fifth experiment of this type, the effect of dividing the nerve was not convincing.

(d) Effects of Various Drugs upon the Rise in Intra-ocular Pressure produced by Stimulation of the Facial Nerve

In the course of the experiments described above various drugs were injected. Flaxedil, as already mentioned, was given in some animals to 18 
eliminate all possibility that striated muscular activity played a part in the production of the rise in intra-ocular pressure. As already stated the rise in pressure was not reduced by Flaxedil; in fact, it seemed that sometimes the effect was potentiated by Flaxedil.

Atropin was injected intravenously in a dosage of $1 \mathrm{mg} . / \mathrm{kg}$. body weight in three cats and five rabbits. In one cat, atropin caused a definite reduction of the rise in intra-ocular pressure, while in the other two cats there was only a slight diminution of the response; in all five animals lacrimation and salivation were not produced by stimulation after atropin had been given. In one of the five rabbits, lacrimation and salivation occurred during stimulation after atropin and it was concluded that this animal was atropinresistant. In the four remaining rabbits, atropin completely abolished both lacrimation and salivation, but the effect upon the rise in intra-ocular pressure was variable; in one rabbit there was a definite reduction in the rise, in another there was a slight diminution, and in the two remaining rabbits there was no change in the response. Despite this variability it can be said with certainty that the effect was not abolished by atropin.

Eserine was injected intravenously in a dosage of $0.5 \mathrm{mg} . / \mathrm{kg}$. in four rabbits. Again, the effect of the drug was variable; in one animal the rise in intra-ocular pressure was definitely abolished, and in the remaining three rabbits the response was unchanged. The response was certainly not potentiated by eserine.

Dibenylene $\left(3 \mathrm{mg} . / \mathrm{kg}\right.$.) was administered intravenously in one cat ${ }^{\circ}$ and, despite the large reduction in systemic blood pressure caused by this drug, the rise in intra-ocular pressure evoked by stimulation of the facial nerve was clearly unaffected.

In two rabbits, acetazoleamide sodium (Diamox) injected intravenously in a dosage of $50 \mathrm{mg} . / \mathrm{kg}$. was found to have no effect upon the response of the intra-ocular pressure.

\section{Discussion}

These experiments have shown that the electrical stimulation of the facial nerve frequently causes a rise in intra-ocular pressure in anaesthetized cats and rabbits. When the stimulus is applied to the greater superficial petrosal nerve, the rise in pressure is confined to the ipsilateral eye. When the stimulus is applied to the fibres of the facial nerves of the two sides simultaneously by placing the electrode in the floor of the fourth ventricle in the mid-line, then a bilateral rise in intra-ocular pressure is obtained. It is clear that the rise in pressure does not result from muscular pressure on the eye.

Greaves and Perkins (1956) stimulated the facial nerves in monkeys but did not find any change in intra-ocular pressure apart from that which could be attributed to pressure on the globe from contraction of extra-ocular muscles. It is possible that the effect occurs in certain species only, although in the present investigation, the responses found in cats were very similar 
to those found in rabbits; apart from this, the present series of experiments differed from those of Greaves and Perkins in certain respects. The occurrence of lacrimation was frequent in the experiments described above, providing some indication that parasympathetic pathways running to the ocular region were activated adequately, but lacrimation was not reported by Greaves and Perkins. Furthermore, it is to be noted that in the experiments of these workers the cervical sympathetic ganglia had been extirpated several weeks previously and that striated muscular activity was eliminated by the injection of decamethonium iodide, a substance which is known to liberate histamine; thus the state of the intra-ocular vascular circulation may have been quite different from that obtaining in the present experiments.

In considering the nervous pathways involved in rises in intra-ocular pressure described above, there are three possibilities:

(i) That the effect was mediated by efferent fibres in the facial nerve,

(ii) That impulses passed up afferent fibres in the facial nerve and that some other efferent nervous pathway was activated reflexly,

(iii) That there was a minimal spread of the stimulus to some other nervous structure.

The first of these three possibilties is the only one which can be accepted, since it was found that rises in intra-ocular pressure obtained in rabbits by stimulation in the floor of the fourth ventricle could be abolished by destroying the facial nerve at the origin of the greater superficial petrosal nerve. Thus it appears that the facial nerve contains efferent fibres which on stimulation cause a rise in intra-ocular pressure. This view is supported by the observations made on cats, that rises in intra-ocular pressure evoked by diencephalic stimulation and believed to be of vascular causation were eliminated by division of the facial nerve at the internal auditory meatus.

Furthermore, the efferent fibres mediating the rise in intra-ocular pressure must leave the main trunk of the facial nerve in the greater superficial petrosal nerve because in the majority of experiments all branches of the main trunk distal to the origin of the petrosal nerve had been divided and the main trunk itself had been cut at the stylo-mastoid foramen. The greater superficial petrosal nerve is known to carry some of the parasympathetic outflow of the facial nerve; after leaving the main trunk of the nerve it runs through a small canal in the tip of the petrous temporal bone, joins with the deep petrosal nerve to form the Vidian nerve (nerve of the pterygoid canal), passes to the sphenopalatine ganglion, and is thence distributed, amongst other destinations, to the lacrimal gland. In the great majority of these experiments, if a rise in pressure occurred in an eye, then there was also lacrimation on that side. It is clear, therefore, quite apart from consideration of the changes in intra-ocular pressure, that the nervous pathway stimulated must include intra-orbital structures in its peripheral distribution. 
Perhaps the most important feature of the responses described here is that the rise in intra-ocular pressure often persisted without diminution for the entire period of stimulation. Thus, Figs 3 and 4 show rises in intra-ocular pressure which were maintained undiminished during stimulations lasting 5 and 16 minutes respectively, and Figs 8 and 9 show responses in which the pressure in the eye rose gradually over periods of 5 and 7 minutes respectively, the full effect obviously not having been reached at the cessation of stimulation. A rise in intra-ocular pressure induced by external pressure on the globe would be quite different in character; one would expect a fairly rapid rise followed by a slow decline towards the initial level. Apart from this consideration, the finding that the rise in intra-ocular pressure was undiminished after the administration of Flaxedil proved that striated muscular activity played no part in the response. In cats, a contraction of the orbital smooth muscle could cause a temporary rise in intra-ocular pressure, but stimulation was not accompanied by retraction of the nictitating membrane, nor was the response impaired after the administration of dibenylene which would block the adrenergic innervation of smooth muscle.

A possible indication of the underlying mechanism of the response is given by the course in time of the rise in intra-ocular pressure, in that there was often an initial rapid rise followed by a more gradual rise. It is possible to attribute these two phases of the response to a single basic effect, viz. a dilatation of intra-ocular blood vessels; the initial rapid rise would be a manifestation of the intra-ocular volumetric changes due to vasodilatation, while the succeeding slow rise would be attributable to an increased rate of aqueous formation consequent upon dilatation of the blood vessels of the ciliary body. It has been shown by Chorobski and Penfield (1932), Forbes, Nason, Cobb, and Wortman (1937), and Greaves and Perkins (1956) that stimulation of the facial nerve produces dilatation of the blood vessels of the pia mater, and it is conceivable that this effect is also exerted upon the vessels of the uvea, a tissue which stands in a close relationship developmentally with the pia mater. It should be added that the increase in formation of aqueous humour postulated above is to be regarded as a direct result of vasodilatation; that is to say, there is an increase in that part of the aqueous formation which is dependent upon hydrostatic forces. The possibility that there is augmentation of the secretory activity of the ciliary body is less likely, since the rise in intra-ocular pressure was not diminished by the administration of acetazoleamide which is believed to reduce the active secretion of the ciliary processes.

Various drugs were used in order to determine whether the nerve-endings involved in this response of the intra-ocular pressure were adrenergic or cholinergic. The persistence of the response after the administration of dibenyelene rules out the possibility that an adrenergic mechanism is involved. The effect of atropin upon the response was variable; in some animals there was a slight reduction of the effect, in other animals the rise 
in intra-ocular pressure was undiminished after atropin. It should be emphasized that, in four out of five rabbits to which atropin was administered, the lacrimation which accompanied the rise in intra-ocular pressure was abolished. This observation proves two points: first, that most of the rabbits were not atropin-resistant, and second that the dosage of atropin was large enough to inhibit cholinergic nerve-endings in the lacrimal gland. Despite the variability of the results with atropin, it can be said with certainty that this drug does not inhibit markedly the rise in intra-ocular pressure evoked by stimulation of the facial nerve. The reasons why atropin may fail to inhibit a cholinergic effect were discussed by Ambache (1955), who emphasized that failure to eliminate a nervous effect with atropin did not necessarily disprove the cholinergic nature of the nerve-endings. In this connexion it is interesting to note that a well-known failure of atropin to inhibit a cholinergic effect concerns the facial nerve; stimulation of the chorda tympani causes secretion from a vasodilatation in the submandibular salivary gland, and after giving atropin, the secretion is abolished while the vasodilatation persists (Heidenhain, 1872; Hilton and Lewis, 1955).

In order to examine further the possibility that cholinergic nerve-endings were involved in the production of the rise in intra-ocular pressure during stimulation of the facial nerve, eserine was also used, but again there was some variability; the rise in intra-ocular pressure was completely abolished in one animal but remained unaffected in others. Certainly there was no marked potentiation of the effect as one would expect if a cholinergic mechanism were involved.

In attempting to elucidate the causation of pathologically raised ocular tension in the human, almost every nervous pathway known to enter the orbit has received some attention, and it is not surprising to find that the sphenopalatine (otherwise known as the nasal or Meckel's) ganglion, which receives the fibres of the greater superficial petrosal nerve, has been considered in this respect. Luedde (1912) described three cases under the heading "intraocular tension in glaucoma reduced by anaesthesia of Meckel's ganglion". Post (1921) reported eight cases of glaucoma in which the sphenopalatine ganglion was blocked by cocainization or injection of alcohol, and concluded that it was ". . . definitely established that the intra-ocular tension can be affected by blocking the nerve impulses at the nasal ganglion. . ." Examination of these reports shows that the evidence presented is not convincing but, in view of the findings in the experiments described above, a more critical study of the possibility that the parasympathetic component of the facial nerve influences the pressure in the human eye would appear justifiable.

\section{Summary}

(1) Stimulation of the facial nerve in anaesthetized cats and rabbits causes a rise in the intra-ocular pressure. 
(2) When the stimulus is applied to the origin of the greater superficial petrosal nerve from the main trunk of the facial nerve on one side, the pressure rises in the ipsilateral eye only.

(3) When the stimulus is applied in the floor of the fourth ventricle, where fibres of the seventh nerve from both sides come into juxtaposition, the pressure rises in both eyes.

(4) Rises in intra-ocular pressure induced by stimulation in the diencephalon or in the floor of the fourth ventricle are eliminated by destruction of the facial nerve between the internal auditory meatus and the stylomastoid foramen. Division of the nerve at the stylo-mastoid foramen does not abolish the effect.

(5) It is concluded that the rises in intra-ocular pressure obtained in these experiments were produced by the activation of efferent pathways which run in the seventh nerve but which leave the main trunk of the nerve and pass into the greater superficial petrosal nerve.

(6) The rises in intra-ocular pressure obtained in these experiments frequently persisted without diminution during stimulations of several minutes' duration. Evidence was obtained that neither smooth nor striated muscular activity was responsible for the rise in ocular tension. It is suggested that the effect was due to intra-ocular vasodilatation.

(7) The rises in intra-ocular pressure were present after atropin had been administered, although lacrimation and salivation, which were frequent concomitant effects, were abolished by this drug.

(8) The responses of the intra-ocular pressure were not potentiated by eserine.

I wish to thank Sir Stewart Duke-Elder for his constant encouragement and advice during the course of this investigation. I am grateful also to Miss S. Bull for technical assistance.

\section{REFERENCES}

Ambache, N. (1955). Pharmacol. Rev., 7, 467.

ChOROBSKI, J., and PenfIELD, W. (1932). Arch. Neurol. Psychiat. (Chicago), 28, 1257.

Forbes, H. S., NASON, G. I., CobB, S., and WORTMAN, R. C. (1937). Ibid., 37, 776.

GLOSTER, J. (1959). Docum. ophthal., 13, 118.

- and GreaVes, D. P. (1957). Brit. J. Ophthal., 41, 513.

(1958). Ibid., 42, 385.

Greaves, D. P., and Perkins, E. S. (1956). J. Physiol. (Lond.), 134, 393.

Heidenhain, R. (1872). Pflüg. Arch. ges. Physiol., 5, 309.

Hilton, S. M., and LewIs, G. P. (1955). J. Physiol. (Lond.), 128, 235.

LUEDDE, W. H. (1912). Amer. J. Ophthal., 0.s. 29, 289.

PERKINS, E. S. (1957). Brit. J. Ophthal., 41, 257.

POST, M. H. (1921). Arch. Ophthal. (N.Y.), 50, 317.

von SallmanN, L., and Lowenstern, O. (1955). Amer. J. Ophthal., 39, No. 4, pt. 2, p. 11. 